\title{
REM observations of GRB 060418 and GRB 060607 A: the onset of the afterglow and the initial fireball Lorentz factor determination ${ }^{\star}$
}

\author{
E. Molinari ${ }^{1}$, S. D. Vergani ${ }^{2,3}$, D. Malesani ${ }^{4,5}$, S. Covino ${ }^{1}$, P. D’Avanzo ${ }^{6,1}$, G. Chincarini ${ }^{7,1}$, \\ F. M. Zerbi ${ }^{1}$, L. A. Antonelli ${ }^{8}$, P. Conconi ${ }^{1}$, V. Testa ${ }^{8}$, G. Tosti ${ }^{9}$, F. Vitali ${ }^{8}$, F. D'Alessio ${ }^{8}$, G. Malaspina ${ }^{1}$, L. Nicastro ${ }^{10}$, \\ E. Palazzi ${ }^{10}$, D. Guetta ${ }^{8}$, S. Campana ${ }^{1}$, P. Goldoni ${ }^{11,12}$, N. Masetti ${ }^{10}$, E. J. A. Meurs ${ }^{2}$, A. Monfardini ${ }^{13}$, L. Norci $^{3}$, \\ E. Pian ${ }^{14}$, S. Piranomonte ${ }^{8}$, D. Rizzuto ${ }^{1,7}$, M. Stefanon ${ }^{15}$, L. Stella ${ }^{8}$, G. Tagliaferri ${ }^{1}$, P. A. Ward ${ }^{2}$, G. Ihle ${ }^{15}$, \\ L. Gonzalez ${ }^{15}$, A. Pizarro ${ }^{15}$, P. Sinclaire ${ }^{15}$, and J. Valenzuela ${ }^{15}$
}

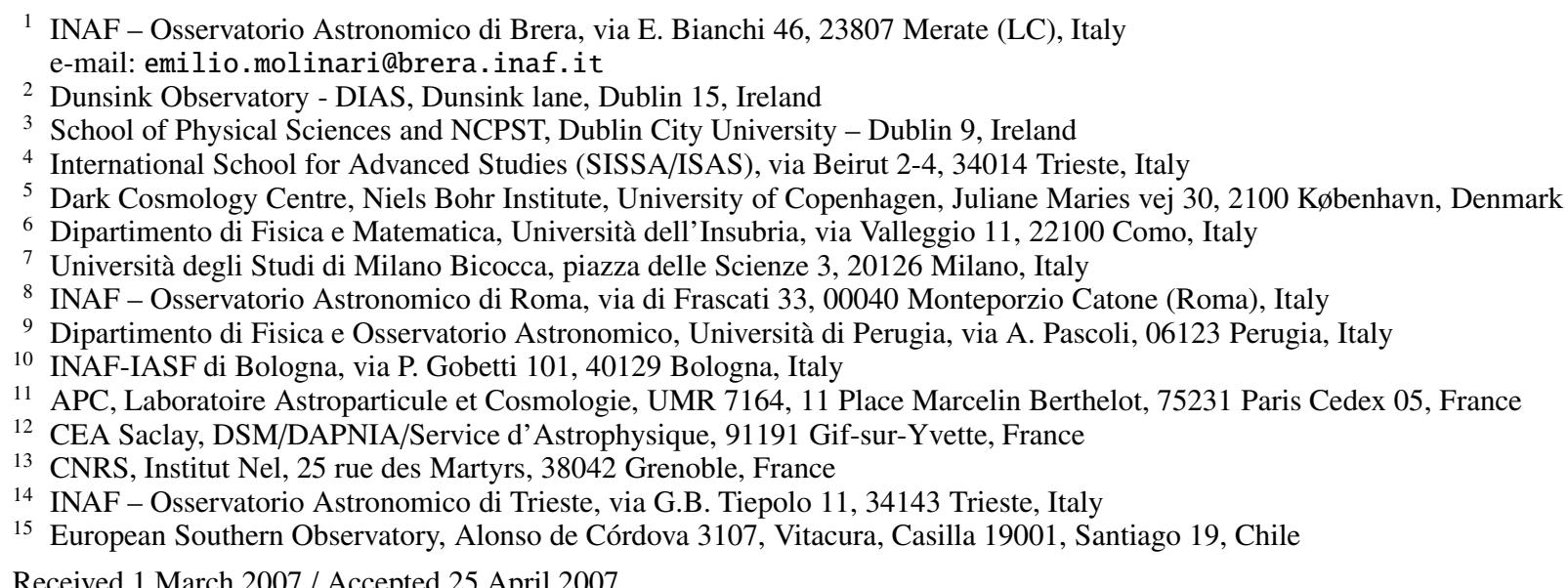

Received 1 March 2007 / Accepted 25 April 2007

\section{ABSTRACT}

\begin{abstract}
Context. Gamma-ray burst (GRB) emission is believed to originate in highly relativistic fireballs.
Aims. Currently, only lower limits were securely set to the initial fireball Lorentz factor $\Gamma_{0}$. We aim to provide a direct measure of $\Gamma_{0}$. Methods. The early-time afterglow light curve carries information about $\Gamma_{0}$, which determines the time of the afterglow peak. We have obtained early observations of the near-infrared afterglows of GRB 060418 and GRB 060607A with the REM robotic telescope. Results. For both events, the afterglow peak could be clearly singled out, allowing a firm determination of the fireball Lorentz of $\Gamma_{0} \sim 400$, fully confirming the highly relativistic nature of GRB fireballs. The deceleration radius was inferred to be $R_{\mathrm{dec}} \approx 10^{17} \mathrm{~cm}$. This is much larger than the internal shocks radius (believed to power the prompt emission), thus providing further evidence for a different origin of the prompt and afterglow stages of the GRB.
\end{abstract}

Key words. gamma rays: bursts - relativity

\section{Introduction}

The early stages of gamma-ray burst (GRB) afterglow light curves display a rich variety of phenomena at all wavelengths and contain significant information which may allow determining the physical properties of the emitting fireball. The launch of the Swift satellite (Gehrels et al. 2004), combined with the development of fast-slewing ground-based telescopes, has hugely improved the sampling of early GRB afterglow light curves. To date there are several published early optical/near-infrared (NIR) afterglow light curves that cannot be fitted with a simple power-law, testifying that the detections started before the afterglow began its regular decay. To explain the early behaviour of most of them (GRB 990123: Akerlof et al. 1999;

* Tables 2 and 3 are only available in electronic form at http: //www . aanda.org
GRB 021004: Kobayashi \& Zhang 2003; GRB 021211: Fox et al. 2003; Li et al. 2003; GRB 041219A: Fan et al. 2005; Vestrand et al. 2005; GRB 050730: Pandey et al. 2006; GRB 050801: Roming et al. 2006; Rykoff et al. 2006; GRB 050820A: Vestrand et al. 2006; GRB 050904: Böer et al. 2006; Wei et al. 2006; GRB 060206: Woźniak et al. 2006; Monfardini et al. 2006; GRB 060210: Stanek et al. 2007) different mechanisms have been proposed, i.e. reverberation of the prompt emission radiation, reverse and refreshed shocks and/or energy injection and, lately, large angle emission (Panaitescu \& Kumar 2007).

Since many processes work in the early afterglow, it is often difficult to model them well enough to be able to determine the fireball characteristics. The simplest case is a light curve shaped by the forward shock only, as could be the case for GRB 030418 (Rykoff et al. 2004) and GRB 060124 (Romano et al. 2006), but 
the lack of a measured redshift in the former case and the poor sampling in the latter prevented to derive firm conclusions. This case is particularly interesting because, while the late-time light curve is independent of the initial conditions (the self-similar solution), the time at which the afterglow peaks depends on the original fireball Lorentz factor $\Gamma$, thus allowing a direct measurement of this fundamental parameter (Sari \& Piran 1999). The short variability timescales, coupled with the nonthermal GRB spectra, indeed imply that the sources emitting GRBs have a highly relativistic motion (Ruderman 1975; Fenimore et al. 1993; Piran 2000; Lithwick \& Sari 2001), to avoid suppression of the high-energy photons due to pair production. This argument, however, can only set a lower limit to the fireball Lorentz factor. Late-time measurements (weeks to months after the GRB) have shown $\Gamma \sim$ a few (Frail et al. 1997; Taylor et al. 2005), but a direct measure of the initial value (when $\Gamma$ is expected to be $\sim 100$ or more) is still lacking.

We present here the NIR early light curves of the GRB 060418 and GRB 060607A afterglows observed with the REM robotic telescope ${ }^{1}$ (Zerbi et al. 2001; Chincarini et al. 2003) located in La Silla (Chile). These afterglows show the onset of the afterglow and its decay at NIR wavelengths as simply predicted by the fireball forward shock model, without the presence of flares or other peculiar features.

\section{Data}

GRB 060418 and GRB 060607A were detected by Swift at 03:06:08 UT (Falcone et al. 2006a) and 05:12:13 UT (Ziaeepour et al. 2006), respectively. The BAT light curve of the former $\left(T_{90}=52 \pm 1 \mathrm{~s}\right)$ showed three overlapping peaks (Cummings et al. 2006). For the latter, the light curve is dominated by a double-peaked structure with a duration $T_{90}=100 \pm 5 \mathrm{~s}$ (Tueller et al. 2006). The Swift XRT started observing the fields 78 and $65 \mathrm{~s}$ after the trigger, respectively. For both bursts a bright, previously uncatalogued fading source was singled out. The XRT light curves show prominent flaring activity, superposed to the regular afterglow decay. For GRB 060418 a prominent peak, also visible in the BAT data, was observed at about $128 \mathrm{~s}$ after the trigger (Falcone et al. 2006b) while the XRT light curve of GRB 060607A was characterised by at least three flares (Page et al. 2006). UVOT promptly detected bright optical counterparts for both events. The redshift is $z=1.489$ for GRB 060418 (Dupree et al. 2006; Vreeswijk \& Jaunsen 2006) and $z=3.082$ for GRB 060607A (Ledoux et al. 2006).

The REM telescope reacted promptly to both GCN alerts and began observing the field of GRB $06041864 \mathrm{~s}$ after the burst (39s after the reception of the alert) and the field of GRB 060607A 59s after the burst (41 s after the reception of the alert). REM hosts REMIR, an infrared imaging camera that operates in the range 1.0-2.3 $\mu \mathrm{m}\left(z^{\prime} J H K^{\prime}\right)$, and ROSS, an optical imager and slitless spectrograph. For both targets a bright NIR source was identified (Covino et al. 2006a,b). ROSS could not observe these two GRBs due to maintenance work. REM followed the two events down to the sensitivity limits adopting two different observing strategies. In the former case multi-colour $z^{\prime} J H K$-band observations were carried out while in the latter case only the $H$ filter was used in order to get a denser sampling of the light curve in just one band. All our photometric data are reported in Tables 2 and 3 available in electronic form.

\footnotetext{
${ }^{1}$ http://www.rem.inaf.it
}

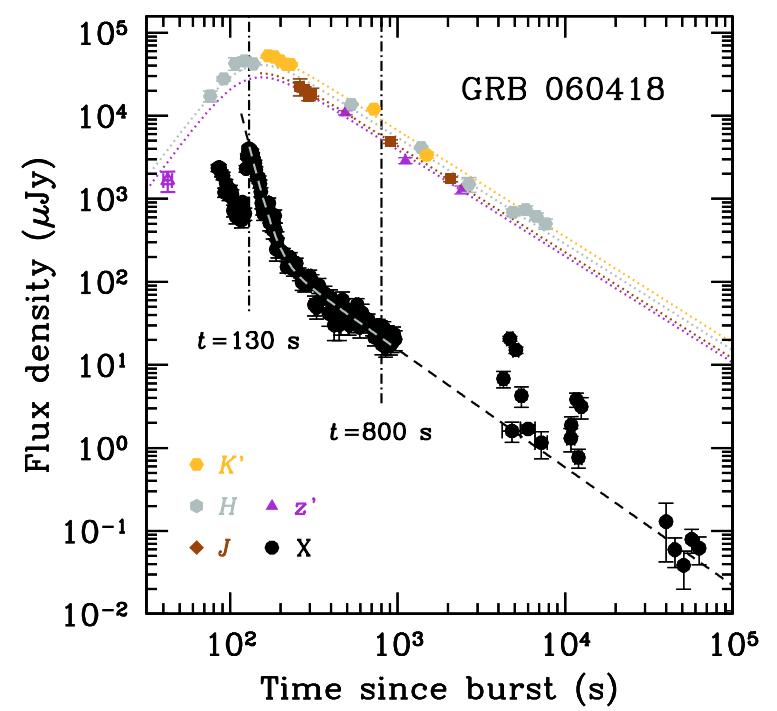

Fig. 1. NIR and X-ray light curves of GRB 060418. The dotted lines show the models of the NIR data using the smoothly broken power law (see Sect. 3.1), while the dashed line shows the best-fit to the X-ray data. The vertical lines mark the epochs at which we computed the SED (Fig. 3).

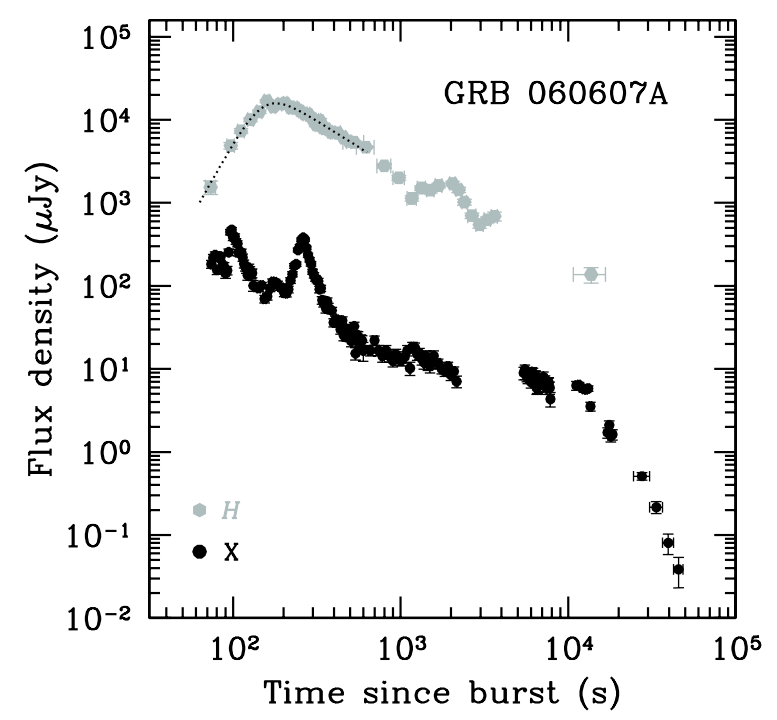

Fig. 2. $H$-band and X-ray light curves of GRB 060607A. The dotted line shows the model to the NIR data using the smoothly broken power law (see Sect. 3.1).

\section{Results and discussion}

\subsection{Light curve modelling}

Figures 1 (GRB 060418) and 2 (GRB 060607A) show the NIR and X-ray light curves of the two afterglows. The X-ray data have been taken with the Swift XRT. The analysis was carried out adopting the standard pipeline. Pile-up corrections both in WT and PC mode were taken into account when required. The NIR light curves of the two events show a remarkable similarity. Both present an initial sharp rise, peaking at 100-200 s after the burst. The NIR flux of GRB 060418 decays afterwards as a regular power law. The NIR light curve of GRB 060607A shows a similar, smooth behaviour up to $\sim 1000 \mathrm{~s}$ after the trigger, followed by a rebrightening lasting $\sim 2000 \mathrm{~s}$.

To quantitatively evaluate the peak time, we fitted the NIR light curves using a smoothly broken power-law 
Table 1. Best fit values of the light curves of the first hour of observations for GRB 060418 and the first $1000 \mathrm{~s}$ for GRB 060607A ( $1 \sigma$ errors), using the smoothly broken power-law reported in Sect. 3.1 with $t_{\text {peak }}=t_{\mathrm{b}}\left(-\alpha_{\mathrm{r}} / \alpha_{\mathrm{d}}\right)^{1 /\left[\kappa\left(\alpha_{\mathrm{d}}-\alpha_{\mathrm{r}}\right)\right]}$. The relatively large $\chi^{2}$ of the fit results from small-scale irregularities present throughout the light curve (see Figs. 1 and 2).

\begin{tabular}{lllllll}
\hline \hline GRB & $t_{\text {peak }}(\mathrm{s})$ & $t_{\mathrm{b}}(\mathrm{s})$ & $\alpha_{\mathrm{r}}$ & $\alpha_{\mathrm{d}}$ & $\kappa$ & $\chi^{2} /$ d.o.f. \\
\hline 060418 & $153_{-10}^{+10}$ & $127_{-21}^{+18}$ & $-2.7_{-1.7}^{+1.0}$ & $1.28_{-0.05}^{+0.05}$ & $1.0_{-0.4}^{+0.4}$ & $33.3 / 16$ \\
$060607 \mathrm{~A}$ & $180_{-6}^{+5}$ & $153_{-12}^{+12}$ & $-3.6_{-1.1}^{+0.8}$ & $1.27_{-0.11}^{+0.16}$ & $1.3_{-1.1}^{+0.9}$ & $28.5 / 19$ \\
\hline
\end{tabular}

(Beuermann et al. 1999): $F(t)=F_{0} /\left[\left(t / t_{\mathrm{b}}\right)^{\kappa \alpha_{\mathrm{r}}}+\left(t / t_{\mathrm{b}}\right)^{\kappa \alpha_{\mathrm{d}}}\right]^{1 / \kappa}$, where $F_{0}$ is a normalisation constant, $\alpha_{\mathrm{r}(\mathrm{d})}$ is the slope of the rise (decay) phase $\left(\alpha_{\mathrm{r}}<0\right)$ and $\kappa$ is a smoothness parameter. The time at which the curve reaches its maximum is $t_{\text {peak }}=t_{\mathrm{b}}\left(-\alpha_{\mathrm{r}} / \alpha_{\mathrm{d}}\right)^{1 /\left[\kappa\left(\alpha_{\mathrm{d}}-\alpha_{\mathrm{r}}\right)\right]}$. We obtain for GRB 060418 and GRB $060607 \mathrm{~A}$ peak times of $153 \pm 10$ and $180 \pm 6 \mathrm{~s}$, respectively. Any other suitable functional forms provides comparable results. The complete set of fit results is reported in Table 1.

As for many other GRBs observed by Swift, the early X-ray light curves of both events show several, intense flares superimposed on the power-law decay (Chincarini et al. 2007). In particular, for GRB 060418 a bright flare was active between $\sim 115$ and $185 \mathrm{~s}$. Excluding flaring times, the decay is then described by a power law with decay slope $\alpha_{\mathrm{X}}=1.42 \pm 0.03$. The X-ray light curve of GRB 060607A is more complex and presents two large flares within the first $400 \mathrm{~s}$. After that the flux density decreases following a shallow power law (with small-scale variability), until steepening sharply at $t \sim 10^{4} \mathrm{~s}$.

By comparing the X-ray and NIR light curves of both bursts, it is apparent that the flaring activity, if any, is much weaker at NIR frequencies. It is thus likely that the afterglow peak, visible in the NIR, is hidden in the X-ray region. In the case of GRB 060418, thanks to our multicolour data, this can be confirmed by considering the NIR/X-ray spectral energy distribution (SED; Fig. 3). At $t=800 \mathrm{~s}$, when both the NIR and $\mathrm{X}$-ray light curves are decaying regularly, the SED is described by a synchrotron spectrum, with the cooling frequency lying at $v_{\text {cool }} \sim 3 \times 10^{15} \mathrm{~Hz}$. The spectral slopes at NIR and X-ray wavelengths are $\beta_{\mathrm{NIR}}=0.65 \pm 0.06$ and $\beta_{\mathrm{X}}=1.03 \pm 0.04$, respectively. A small amount of extinction in the host $\left(A_{\mathrm{V}}=0.10 \mathrm{mag}\right.$ assuming the SMC extinction curve) was considered to make $\beta_{\text {NIR }}$ consistent with the synchrotron expectation $\beta_{\mathrm{NIR}}=\beta_{\mathrm{X}}-0.5$. The situation is clearly different at early times, when $\mathrm{X}$-ray flaring is active: the $\mathrm{X}$-ray component is softer $\left(\beta_{\mathrm{X}}=1.27 \pm 0.03\right)$ and much brighter than the extrapolation of the NIR emission (even including the dust correction). This implies a different origin for the X-ray emission. For GRB 060607A, the light curve is complicated by several flares. As we lack multicolour data, a detailed analysis is not possible.

\subsection{Determination of the Lorentz factor $\Gamma_{0}$}

Our spectral and temporal analysis agrees with the interpretation of the NIR afterglow light curves as corresponding to the afterglow onset, as predicted by the fireball forward shock model (Sari \& Piran 1999; Mészáros 2006). According to the fits, the light curves of the two afterglows peak at a time $t_{\text {peak }}>T_{90}$ as expected in the impulsive regime outflow ("thin shell" case). In this scenario the quantity $t_{\text {peak }} /(1+z)$ corresponds to the deceleration timescale $t_{\mathrm{dec}} \sim R_{\mathrm{dec}} /\left(2 c \Gamma_{\mathrm{dec}}^{2}\right)$, where $R_{\mathrm{dec}}$ is the deceleration radius, $c$ is the speed of light and $\Gamma_{\text {dec }}$ is the fireball

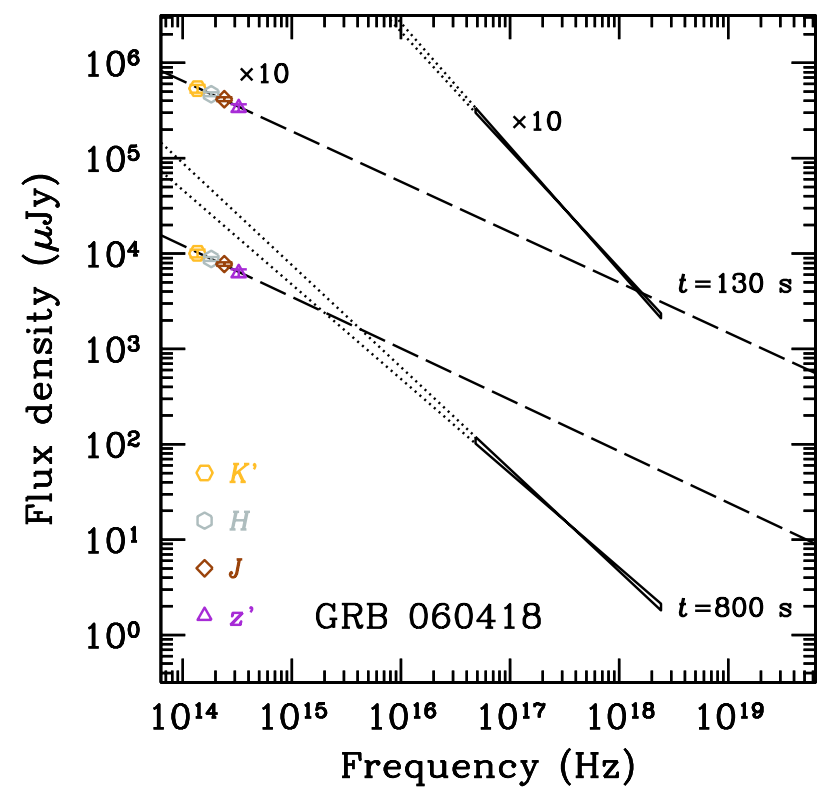

Fig. 3. SED of the GRB 060418 afterglow at 130 and $800 \mathrm{~s}$ after the trigger. NIR data have been corrected for Galactic and host extinction $\left(A_{\mathrm{V}}=0.74\right.$ and $0.10 \mathrm{mag}$, respectively).

Lorentz factor at $t_{\mathrm{dec}}$. It is therefore possible to estimate $\Gamma_{\mathrm{dec}}$ (Sari \& Piran 1999), which is expected to be half of the initial value $\Gamma_{0}$ (Panaitescu \& Kumar 2000; Mészáros 2006). For a homogeneous surrounding medium with particle density $n$, we have

$\Gamma\left(t_{\text {peak }}\right)=\left[\frac{3 E_{\gamma}(1+z)^{3}}{32 \pi n m_{\mathrm{p}} c^{5} \eta t_{\text {peak }}^{3}}\right]^{1 / 8} \approx 160\left[\frac{E_{\gamma, 53}(1+z)^{3}}{\eta_{0.2} n_{0} t_{\text {peak }, 2}^{3}}\right]^{1 / 8}$,

where $E_{\gamma}=10^{53} E_{\gamma, 53} \mathrm{erg}$ is the isotropic-equivalent energy released by the GRB in gamma rays, $n=n_{0} \mathrm{~cm}^{-3}$, $t_{\text {peak }, 2}=t_{\text {peak }} /(100 \mathrm{~s}), \eta=0.2 \eta_{0.2}$ is the radiative efficiency and $m_{\mathrm{p}}$ is the proton mass. We use $E_{\gamma}=9 \times 10^{52} \mathrm{erg}$ for GRB 060418 (Golenetskii et al. 2006) and $E_{\gamma} \sim 1.1 \times 10^{53} \mathrm{erg}$ for GRB 060607A (Tueller et al. 2006). Substituting the measured quantities and normalising to the typical values $n=1 \mathrm{~cm}^{-3}$ and $\eta=0.2$ (Bloom et al. 2003), we infer for both bursts $\Gamma_{0} \approx 400\left(\eta_{0.2} n_{0}\right)^{-1 / 8}$. This value is very weakly dependent on the unknown parameters $n$ and $\eta$, and therefore provides a robust determination of $\Gamma_{0}$.

One possible caveat concerns the density profile of the surrounding medium, which is still uncertain. Chevalier \& $\mathrm{Li}$ (2000) proposed a wind-shaped profile $n(r)=A r^{-2}$, where $A$ is a constant, as expected around a massive star. In principle, the temporal and spectral properties of the afterglow can be used to distinguish among the two cases. For $v<v_{\text {cool }}$, before $t_{\mathrm{dec}}$, the flux is expected to rise as $t^{3}$ for the homogeneous (ISM) case, while it evolves slower than $t^{1 / 3}$ in a wind environment. The measured values for GRB 060418 and GRB 060607A seem therefore consistent with the ISM case (see also Jin \& Fan 2007). For GRB 060418, however, no closure relation is satisfied for $t>t_{\text {peak }}$. In fact, irrespective of the density profile, the temporal and spectral slopes in the X-ray region (which lies above $v_{\text {cool }}$ ) should verify $\alpha_{\mathrm{X}}=3 \beta_{\mathrm{X}} / 2-1 / 2$, which is clearly not the case given the observed values. Such discrepancy has been observed in many other examples and may be due to radiative losses, or varying equipartition parameters, or Compton losses (see e.g. Panaitescu 2005; Zhang et al. 2006). A detailed treatment of 
these effects is beyond the scope of this paper. We however provide an estimate of $\Gamma_{0}$ in the case of a wind environment:

$\Gamma\left(t_{\text {peak }}\right)=\left[\frac{E_{\gamma}(1+z)}{8 \pi A m_{\mathrm{p}} c^{3} \eta t_{\text {peak }}}\right]^{1 / 4}$.

Using the same values as above for $E$ and $z$, and assuming $A^{*}=$ $A /\left(3 \times 10^{35} \mathrm{~cm}^{-1}\right)=1$, we find $\Gamma_{0} \approx 150$ for both bursts.

The determination of $t_{\text {peak }}$ is in principle affected by the choice of the time origin $t_{0}$, which we set to the BAT trigger time (Lazzati \& Begelman 2006; Quimby et al. 2006). These authors have however shown that this effect is small, and mostly affects the rise and decay slopes rather than the peak time (especially when $t_{\text {peak }}$ is larger than the burst duration, as for GRB 060418 and GRB 060607A). The measurement of $\Gamma_{0}$ is thus not very sensitive to the exact choice of $t_{0}$.

For both bursts, we could not detect any reverse shock emission. The lack of such flashes has already been noticed previously in a set of Swift bursts with prompt UVOT observations (Roming et al. 2006). Among the many possible mechanisms to explain the lack of this component, strong suppression (or even total lack) of reverse shock emission is naturally expected if the outflow is Poynting-flux dominated (Fan et al. 2004; Zhang \& Kobayashi 2005). Nevertheless, Jin \& Fan (2007) showed that for GRB 060418 and GRB 060607A the reverse shock emission might be too weak to be detected.

Finally, the optical afterglow of GRB 030418 showed a peaked light curve similar to our cases, and has been explained by Rykoff et al. (2004) as due to decreasing extinction. Our peaks are too sharp to match this interpretation. Furthermore, a very large extinction would be implied, which contrasts with the UVOT detection in the rest-frame UV (Schady et al. 2006; Oates et al. 2006). The peak of GRB 030418 might have been the afterglow onset. Setting $z=1$, we get $\Gamma_{0} \sim 100$ using Eq. (1).

\section{Conclusions}

The REM discovery of the afterglow onset has demonstrated once again the richness and variety of physical processes occurring in the early afterglow stages. The very fast response observations presented here provide crucial information on the GRB fireball parameters, most importantly its initial Lorentz factor. This is the first time that $\Gamma\left(t_{\text {peak }}\right)$ is directly measured from the observations of a GRB. The measured $\Gamma_{0}$ value is well within the range (50 $\left.\lesssim \Gamma_{0} \lesssim 1000\right)$ envisaged by the standard fireball model (Piran 2000; Guetta et al. 2001; Soderberg \& RamirezRuiz 2002; Mészáros 2006). It is also in agreement with existing measured lower limits (Lithwick \& Sari 2001; Zhang et al. 2006). Albeit the values for $\Gamma_{0}$ are comparable for GRB 060418 and GRB 060607A, as noted in the introduction prompt optical detections have shown very different behaviours, so this value should not be taken as common to every GRB.

Using $\Gamma_{0}=400$ we can also derive other fundamental quantities characterising the fireball of the two bursts. In particular, the isotropic-equivalent baryonic load of the fireball is $M_{\mathrm{fb}}=E /\left(\Gamma_{0} c^{2}\right) \approx 7 \times 10^{-4} M_{\odot}$, and the deceleration radius is $R_{\text {dec }} \approx 2 c t_{\text {peak }}\left[\Gamma\left(t_{\text {peak }}\right)\right]^{2} /(1+z) \approx 10^{17} \mathrm{~cm}$. This is much larger than the scale of $\sim 10^{15} \mathrm{~cm}$ where the internal shocks are believed to power the prompt emission (Mészáros \& Rees 1997; Rees \& Mészáros 1994), thus providing further evidence for a different origin of the prompt and afterglow stages of the GRB.

Acknowledgements. We thank Yi-Zhong Fan, Zhi-Ping Jin, Massimo Della Valle and D. Alexander Kann for discussion, and an anonymous referee for suggestions. The REM project has been accomplished thanks to the support of the
Italian Ministry of Education (MURST/COFIN, PI G.C.). Part of the instrumentation has been partially supported by ASI (ROSS spectrograph, PI E.Pa.), by CNAA and by the Swift Project (supported by ASI); present maintenance and operation of the REM telescope is supported by INAF (PI E.M.). We also acknowledge the sponsorship of the Italian division of AMD who provided computers for the REM observatory. REM is the result of a collaboration between a group of Italian research institutes, coordinated by the Osservatorio Astronomico di Brera, the Laboratoire Astroparticule et Cosmologie and the DSM/DAPNIA/Service d'Astrophysique (France), the Dunsink Observatory and UCD (Ireland). S.D.V. and D.M. are supported by SFI and IDA, respectively.

\section{References}

Akerlof, C., Balsano, R., Barthelmy, S., et al. 1999, Nature, 398, 400 Beuermann, K., Hessman, F. V., Reinsch, K., et al. 1999, A\&A, 352, L26 Böer, M., Atteia, J. L., Damerdji, Y., et al. 2006, ApJ, 638, 71 Bloom, J. S., Frail, D. A., \& Kulkarni, S. R. 2003, ApJ, 594, 674 Chevalier, R. A., \& Li, Z.-Y. 2000, ApJ, 536, 195

Chincarini, G., Zerbi, F. M., Antonelli, A., et al. 2003, The Messenger, 113, 40 Chincarini, G., Moretti, A., Romano, P., et al. 2007, ApJ, submitted [arXiv: astro-ph/0702371]

Covino, S., Antonelli, L. A., Vergani, S. D., et al. 2006a, GCN, 4967 Covino, S., Distefano, E., Molinari, E., et al. 2006b, GCN, 5234 Cummings, J., Barbier, L., Barthelmy, S., et al. 2006, GCN, 4975 Dupree, A. K., Falco, E., Prochaska, J. X., et al. 2006, GCN, 4969 Falcone, A. D., Barthelmy, S. D., Burrows, D. N., et al. 2006a, GCN, 4966 Falcone, A. D., Burrows, D. N., Kennea, J., et al. 2006b, GCN, 4973 Fan, Y. Z., Wei, D. M., \& Wang, C. F. 2004, A\&A, 424, 477 Fan, Y. Z., Zhang, B., \& Wei, D. M. 2005, ApJ, 628, L25 Fenimore, E. E., Epstein, R. I., \& Ho, C. 1993, A\&AS, 97, 59 Fox, D. W., Price, P. A., Soderberg, A. M., et al. 2003, ApJ, 586, L5 Frail, D. A., Kulkarni, S. R., Nicastro, L., Feroci, M., \& Taylor, G. B. 1997, Nature, 389, 261

Gehrels, N., Chincarini, G., Giommi, P., et al. 2004, ApJ, 611, 1005

Golenetskii, S., Aptekar, R., Mazets, E., et al. 2006, GCN, 4989

Guetta, D., Spada, M., \& Waxman, E. 2001, ApJ, 557, 399

Kobayashi, S., \& Zhang, B. 2003, ApJ, 582, 75

Jin, Z.-P., \& Fan, Y.-Z. 2007, MNRAS, accepted [arXiv:astro-ph/0701715] Lazzati, D., \& Begelman, M. C. 2006, ApJ, 641, 972

Ledoux, C., Vreeswijk, P., Smette, A., Jaunsen, A., \& Kaufer, A. 2006, GCN, 5237

Li, W., Filippenko, A. V., Chornok, R., \& Jha, S. 2003, ApJ, 586, L9

Lithwick, Y., \& Sari, R. 2001, ApJ, 555, 540

Mészáros, P. 2006, Rep. Progr. Phys., 69, 2259

Mészáros, P., \& Rees, M. J. 1997, ApJ, 476, 232

Monfardini, A., Kobayashi, S., Guidorzi, C., et al. 2006, ApJ, 648, 1125

Nysewander, M., Ivarsen, K., Foster, A., et al. 2006, GCN, 4971

Oates, S. R., Blustin, A. J., Ziaeepour, H. Z., et al. 2006, GCN, 5243

Page, K., Goad, M., Beardmore, A., et al. 2006, GCN, 5240

Panaitescu, A. 2005, MNRAS, 363, 1409

Panaitescu, A., \& Kumar, P. 2000, ApJ, 543, 66

Panaitescu, A., \& Kumar, P. 2007, MNRAS, 163, 1065

Pandey, S. B., Castro-Tirado, A. J., McBreen, S., et al. 2006, A\&A , 460, 415

Piran, T. 2000, Phys. Rep., 333, 529

Quimby, R., Rykoff, E. S., Yost, S. A., et al. 2006, ApJ, 640, 402

Rees, M. J., \& Mészáros, P. 1994, ApJ, 430, L93

Romano, P., Campana, S., Chincarini, G., et al. 2006, A\&A, 456, 917

Roming, P. W. A., Schady, P., Fox, D. B., et al. 2006, ApJ, 652, 1416

Ruderman, M. 1975, NYASA, 262, 164

Rykoff, E. S., Smith, D. A., Price, P. A., et al. 2004, ApJ, 601, 1013

Rykoff, E. S., Mangano, V., Yost, S. A., et al. 2006, ApJ, 638, 5

Sari, R., \& Piran, T. 1999, ApJ, 520, 641

Schady, P., \& Falcone, A. D. 2006, GCN, 4978

Soderberg, A. M., \& Ramirez-Ruiz, E. 2002, MNRAS, 330, L24

Stanek, K. Z., Dai, X., Prieto, J. L., et al. 2007, ApJ, 654, L21

Taylor, G. B., Momjian, E., Pihlstrom, Y., Ghosh, T., \& Salter, C. 2005, ApJ, 622,986

Tueller, J., Barbier, L., Barthelmy, S., et al. 2006, GCN, 5242

Vestrand, W. T., Wozniank, P. R., Wren, J. A., et al. 2005, Nature, 435, 178

Vestrand, W. T., Wren, J. A., Wozniak, P. R., et al. 2006, Nature, 442, 172

Vreeswijk, P., \& Jaunsen, A. 2006, GCN, 4974

Wei, D. M., Yan, T., \& Fan, Y. Z. 2006, ApJ, 636, 69

Woźniak, P. R., Vestrand, W. T., Wren, J. A., et al. 2006, ApJ, 642, L99

Zerbi, F. M., Chincarini, G., Ghisellini, G., et al. 2001, AN, 322, 275

Zhang, B., \& Kobayashi, S. 2005, ApJ, 628, 315

Zhang, B., Fan, Y. Z., Dyks, J., et al. 2006, ApJ, 642, 354

Ziaeepour, H. Z., Barthelmy, S. D., Gehrels, N., et al. 2006, GCN, 5233 
E. Molinari et al.: The onset of the afterglow, Online Material $p 1$

\section{Online Material}


E. Molinari et al.: The onset of the afterglow, Online Material $p 2$

Table 2. Observation log for GRB 060418. The time $t_{0}$ indicates the BAT trigger time, 2006 April 18.12926 UT. * Nysewander et al. (2006).

\begin{tabular}{lcclc}
\hline $\begin{array}{l}\text { Mean time } \\
\text { (UT) }\end{array}$ & $\begin{array}{c}t-t_{0} \\
(\mathrm{~s})\end{array}$ & $\begin{array}{c}\text { Exp. time } \\
(\mathrm{s})\end{array}$ & Filter & Magnitude \\
\hline Apr. 18.12974 & 40 & 5 & $z$ & $15.3 \pm 0.3^{*}$ \\
Apr. 18.13371 & 479 & 100 & $z^{\prime}$ & $13.26 \pm 0.06$ \\
Apr. 18.14215 & 1114 & 100 & $z^{\prime}$ & $14.70 \pm 0.09$ \\
Apr. 18.15722 & 2416 & 100 & $z^{\prime}$ & $15.58 \pm 0.12$ \\
\hline Apr. 18.13264 & 292 & 100 & $J$ & $12.33 \pm 0.05$ \\
Apr. 18.13975 & 906 & 150 & $J$ & $13.82 \pm 0.06$ \\
Apr. 18.15323 & 2071 & 300 & $J$ & $14.94 \pm 0.07$ \\
\hline Apr. 18.13014 & 76 & 10 & $H$ & $11.98 \pm 0.16$ \\
Apr. 18.13032 & 92 & 10 & $H$ & $11.46 \pm 0.07$ \\
Apr. 18.13050 & 107 & 10 & $H$ & $11.00 \pm 0.19$ \\
Apr. 18.13067 & 122 & 10 & $H$ & $10.92 \pm 0.12$ \\
Apr. 18.13085 & 137 & 10 & $H$ & $11.01 \pm 0.02$ \\
Apr. 18.13538 & 529 & 150 & $H$ & $12.24 \pm 0.03$ \\
Apr. 18.14527 & 1383 & 150 & $H$ & $13.57 \pm 0.04$ \\
Apr. 18.16008 & 2663 & 150 & $H$ & $14.89 \pm 0.22$ \\
Apr. 18.18578 & 4883 & 150 & $H$ & $15.48 \pm 0.10$ \\
Apr. 18.19649 & 5809 & 150 & $H$ & $15.40 \pm 0.12$ \\
Apr. 18.20720 & 6734 & 150 & $H$ & $15.60 \pm 0.10$ \\
Apr. 18.21791 & 7659 & 150 & $H$ & $15.83 \pm 0.15$ \\
\hline Apr. 18.13121 & 168 & 10 & $K^{\prime}$ & $10.23 \pm 0.04$ \\
Apr. 18.13139 & 184 & 10 & $K^{\prime}$ & $10.26 \pm 0.03$ \\
Apr. 18.13157 & 200 & 10 & $K^{\prime}$ & $10.38 \pm 0.03$ \\
Apr. 18.13175 & 215 & 10 & $K^{\prime}$ & $10.48 \pm 0.05$ \\
Apr. 18.13192 & 230 & 10 & $K^{\prime}$ & $10.49 \pm 0.05$ \\
Apr. 18.14652 & 1491 & 300 & $K^{\prime}$ & $13.14 \pm 0.09$ \\
\hline & & & &
\end{tabular}

Table 3. Observation log for GRB 060607A. The time $t_{0}$ indicates the BAT trigger time, 2006 June 7.21682 UT.

\begin{tabular}{lcccc}
\hline \hline Mean time & $\begin{array}{c}t-t_{0} \\
(\mathrm{~s})\end{array}$ & $\begin{array}{c}\text { Exp. time } \\
\text { (s) }\end{array}$ & Filter & Magnitude \\
\hline Jun. 7.21767 & 73 & 10 & $H$ & $14.60 \pm 0.20$ \\
Jun. 7.21794 & 97 & 10 & $H$ & $13.35 \pm 0.10$ \\
Jun. 7.21811 & 111 & 10 & $H$ & $12.91 \pm 0.07$ \\
Jun. 7.21829 & 127 & 10 & $H$ & $12.57 \pm 0.06$ \\
Jun. 7.21848 & 143 & 10 & $H$ & $12.33 \pm 0.05$ \\
Jun. 7.21866 & 159 & 10 & $H$ & $12.01 \pm 0.05$ \\
Jun. 7.21883 & 174 & 10 & $H$ & $12.18 \pm 0.05$ \\
Jun. 7.21902 & 190 & 10 & $H$ & $12.10 \pm 0.05$ \\
Jun. 7.21919 & 205 & 10 & $H$ & $12.07 \pm 0.05$ \\
Jun. 7.21937 & 220 & 10 & $H$ & $12.21 \pm 0.05$ \\
Jun. 7.21955 & 236 & 10 & $H$ & $12.21 \pm 0.05$ \\
Jun. 7.21973 & 251 & 10 & $H$ & $12.31 \pm 0.05$ \\
Jun. 7.21991 & 267 & 10 & $H$ & $12.40 \pm 0.05$ \\
Jun. 7.22008 & 282 & 10 & $H$ & $12.40 \pm 0.05$ \\
Jun. 7.22027 & 298 & 10 & $H$ & $12.55 \pm 0.06$ \\
Jun. 7.22045 & 314 & 10 & $H$ & $12.72 \pm 0.06$ \\
Jun. 7.22062 & 328 & 10 & $H$ & $12.59 \pm 0.06$ \\
Jun. 7.22080 & 344 & 10 & $H$ & $12.82 \pm 0.07$ \\
Jun. 7.22098 & 359 & 10 & $H$ & $12.84 \pm 0.07$ \\
Jun. 7.22128 & 385 & 30 & $H$ & $12.95 \pm 0.05$ \\
Jun. 7.22169 & 421 & 30 & $H$ & $12.96 \pm 0.05$ \\
Jun. 7.22209 & 455 & 30 & $H$ & $13.10 \pm 0.05$ \\
Jun. 7.22251 & 492 & 30 & $H$ & $13.23 \pm 0.05$ \\
Jun. 7.22292 & 527 & 30 & $H$ & $13.25 \pm 0.05$ \\
Jun. 7.22400 & 620 & 150 & $H$ & $13.40 \pm 0.04$ \\
Jun. 7.22606 & 798 & 150 & $H$ & $13.96 \pm 0.06$ \\
Jun. 7.22812 & 976 & 150 & $H$ & $14.32 \pm 0.07$ \\
Jun. 7.23033 & 1167 & 150 & $H$ & $14.94 \pm 0.08$ \\
Jun. 7.23223 & 1331 & 150 & $H$ & $14.63 \pm 0.07$ \\
Jun. 7.23427 & 1508 & 150 & $H$ & $14.69 \pm 0.08$ \\
Jun. 7.23635 & 1687 & 150 & $H$ & $14.55 \pm 0.07$ \\
Jun. 7.24047 & 2043 & 150 & $H$ & $14.49 \pm 0.07$ \\
Jun. 7.24253 & 2221 & 150 & $H$ & $14.68 \pm 0.08$ \\
Jun. 7.24459 & 2399 & 150 & $H$ & $15.05 \pm 0.09$ \\
Jun. 7.24767 & 2665 & 300 & $H$ & $15.46 \pm 0.08$ \\
Jun. 7.25113 & 2964 & 300 & $H$ & $15.73 \pm 0.10$ \\
Jun. 7.25493 & 3293 & 300 & $H$ & $15.58 \pm 0.09$ \\
Jun. 7.25886 & 3632 & 150 & $H$ & $15.48 \pm 0.13$ \\
Jun. 7.37574 & 13730 & 6000 & $H$ & $17.23 \pm 0.23$ \\
\hline & & & &
\end{tabular}

